\title{
Electrophysiological Grading to Assess the Severity of Carpal Tunnel Syndrome in Symptomatic Diabetic Patients
}

\author{
RUMANA HABIB ${ }^{1}$, DILRUBA ALAM ${ }^{2}$, RAFI NAZRUL ISLAM ${ }^{3}$, RASHEDUL ISLAM $^{4}$, \\ NIRMALENDU BIKASH BHOWMIK ${ }^{5}$, ANISUL HAQUE ${ }^{6}$
}

\begin{abstract}
:
Background: Carpal tunnel syndrome (CTS) is the most frequent compressive focal mononeuropathy found in clinical practice. Patients mostly experience pain, paresthesia, and less commonly, weakness in the median nerve distribution which badly affects dexterity and grip. Common risk factors include repetitive wrist movements, metabolic and degenerative diseases, connective tissue disease and pregnancy. Nerve conduction study is one of the most sensitive and specific tools to diagnose CTS. This study was aimed to grade the CTS cases according to the severity based on electrophysiological findings and explore its association with clinical presentation of diabetic patients had nerve conduction study at BIRDEM General Hospital. Materials and Methods: This observational study was done in the electro diagnostic clinic of Neurology department, BIRDEM during the period Sept 2015 to Feb 2016. The study included 100 hands of 84 patients suffering from CTS consecutively attending the clinic. All the patients were interviewed and clinically examined. Demographic data including age, gender, occupation, affected hand and hand dominance along with duration of disease were recorded. Patients were graded according to clinical history and objective findings and again based on the Canterbury NCS Severity Scale. Results: Of these 84 diabetic patients presenting with impression of CTS $96 \%$ were females. Mean age of the study subjects was $49.6 \pm 10.1$ (28-85). Most of the female patients were housewives. Clinical grading of CTS was as follows: mild symptoms in $54.76 \%$, moderate symptoms in $23.8 \%$ and severe symptoms in $28.58 \%$ patients. According to The Canterbury NCS Severity Scale out of total 100 hands, 3.5\% had Grade 2 (Mild) 30.5\%had Grade 3 (Moderate) ,29.4\% had Grade 4 (Severe), 34.1\% had Grade 5 (Very severe) disease. Only 2 patients had Grade 6 (extremely severe) lesion. Of the study subjects 22 (26.19\%) had bilateral and rest (73.81\%) had unilateral disease. Conclusion: Data demonstrated female preponderance of the diabetic CTS cases of middle age. Proportional graded deterioration of electrophysiological parameters along with the clinical severity grades highlights the fact that NCS provide additional and independent objective evidence in the diagnosis and severity assessment of CTS and plausibly has important role in prioritizing treatment plan.
\end{abstract}

Keywords: Carpal Tunnel Syndrome, Nerve Conduction Studies, Canterbury NCS Severity Scale, Diabetes Mellitus

\section{Introduction:}

Carpal Tunnel Syndrome (CTS) is the entrapment of the median nerve within the carpal tunnel. In the carpal tunnel the median nerve lies immediately beneath the Palmaris Longus tendon and anterior to the flexor tendons ${ }^{1}$. Certain conditions, such as diabetes mellitus, amyloidosis, hypothyroidism, and rheumatoid arthritis, obesity, pregnancy can predispose to CTS. ${ }^{2}$. CTS is the most frequent entrapment neuropathy causing

1. Assistant Professor, Department of Neurology, BIRDEM General Hospital, Dhaka

2. Registrar, Department of Neurology, BIRDEM General Hospital, Dhaka

3. Assistant Registrar, Department of Nephrology, BIRDEM General Hospital.

4. Assistant Professor, Department of Neurology,BIRDEM General Hospital, Dhaka

5. Professor, Department ofNeurology, BIRDEM General Hospital, Dhaka

6. Professor and Honorary Senior Consultant, Department ofNeurology, BIRDEM General Hospital, Dhaka 
numbness, tingling, discomfort, pain and weakness in hands ranging from mild to debilitating extent, especially for those whose work or recreational activities require extensive use of hands ${ }^{3}$.

Electro diagnostic (EDx) studies are a valid and reliable means of confirming the clinical diagnosis of CTS . The amplitudes along with the conduction velocities of the sensory nerve action potential and motor nerve action potential reflect the functional state of axons, and are useful parameters and complement the clinical grading in the assessment of severity of CTS ${ }^{4}$. The management of patients diagnosed with CTS is based upon the acuity and severity of clinical symptoms and the degree of neurogenic injury as assessed by electro diagnostic studies and this leads to better management of disease whether by ergonomic modifications, conservative methods or surgical interventions ${ }^{5,6}$.

An audit carried out on records and notes of electrophysiological lab of National Institute of Neurosciences and Hospital (NINS) from January to December 2013 showedCarpal tunnel syndrome (CTS) was the most common condition (19.2\%) observed at the $\mathrm{lab}^{6}$.Common risk factors associated with carpal tunnel syndrome in Bangladesh were identified ${ }^{7}$.Another randomized controlled trial evaluated the efficacy of local corticosteroid in idiopathic carpal tunnel syndrome among 60 idiopathic CTS patients ${ }^{8}$. But assessing the severity of CTS in diabetic patients and grading it on the basis of electro diagnostic studies has not yet been conducted in Bangladesh.

The current study looks at the demographic profile, clinical presentation and the pattern of severity of Carpal Tunnel Syndrome (CTS) in diabetic patients referred for electro diagnostic studies (NCS) at BIRDEM General Hospital, Dhaka.

\section{Methods:}

This is a retrospective analytic electrophyisologic study performed in 100 hands of 85 diabetic patients clinically diagnosed with CTS at a referral hospital in Dhaka. Adult patients aged e" 18 years of both sexes were consecutively and purposively selected from between September, 2015 and February , 2016 for a period of Six (6) months. A thorough medical record review was applied and patients with traumatic median nerve injury, polyneuropathy, history of malignancy, pregnancy, obesity ,hypothyroidism, rheumatoid arthritis and those who have been previously operated for CTS were excluded. The exclusion criteria also included cervical spine related problem. Demographic data including age, gender, occupation , affected hand and hand dominance along with duration of disease were recorded. Patients were graded according to clinical history and objective findings and again based on the Canterbury electrophysiological grading scale.

\section{Clinical grading of CTS severity:}

-CTS is considered mild if there is numbness, tingling, or discomfort in the median nerve distribution but no sensory loss or weakness, no sleep disruption, and no difficulty with hand function or interference with activities of daily living (ADLs).

-CTS is considered moderate if there is sensory loss in the median distribution, or if nocturnal symptoms occasionally disrupt sleep. Symptoms (sensory loss or pain) may interfere slightly with hand function but the patient should be able to perform all ADLs.

-CTS is considered severe if there is weakness in the median distribution, or if symptoms are disabling and prevent the patient from carrying out one or more ADLs, or if nocturnal symptoms routinely disrupt sleep.

NCS was performed by the neurologist with standard surface stimulation and recording techniques. Motor and sensory studies were performed for the ulnar and median nerves. The Sensory component of each nerve was stimulated antidromically while the motor part was stimulated orthodromically and the $F$ wave was recorded. The action potentials was recorded as sensory nerve action potential (SNAP) and compound muscle action potential (CMAP) for sensory and the motor nerves respectively. The parameters obtained were; onset latency, amplitude of CMAP, duration, area, distance and nerve conduction velocity (CV). If the median nerve sensory NCS results were normal,a comparison test was performed to compare sensory conduction values of the median nerve and ulnar nerve between the wrist and ring finger.

CTS was classified into six grades based on the Canterbury electrophysiologic grading scale. The scale grades neurophysiological severity of CTS from 0 - no abnormality, to 6 - extremely severe CTS. 
Electro diagnostic grading of CTS is as follows: normal (grade 0)

very mild (grade 1): CTS demonstrable only with most sensitive tests;

mild (grade 2): sensory nerve conduction velocity slow on finger/wrist measurement, normal terminal motor latency;

moderate (grade 3): sensory potential preserved with motor slowing, distal motor latency to abductor pollicisbrevis (APB) < $6.5 \mathrm{~ms}$;

severe (grade 4): sensory potentials absent but motor response preserved, distal motor latency to APB $<6.5 \mathrm{~ms}$;

very severe (grade 5): terminal latency to APB > $6.5 \mathrm{~ms}$;

extremely severe (grade 6): sensory and motor potentials effectively unrecordable (surface motor potential from APB $<0.2 \mathrm{mV}$ amplitude).

Approval from hospital ethical committee was taken. Verbal informed consent was taken after explaining the purpose of study and use of data for research and publication. A statistical software (SPSS Inc, version 20) was used for analysis. Frequencies and descriptive statistics were calculated for various variables. Descriptive statistics were used to calculate mean and SD for age. The data are presented as tables and figures.

\section{Results:}

This is retrospective analytic study in 100 hands of 84 diabetic patients presented with symptoms and signs of CTS confirmed by NCS. Female preponderance (male 3 vs female 82 ) was present among the 84 study subjects .

Table-I

Age distribution of study population $(N=85)$

\begin{tabular}{lcc}
\hline Age groups & Frequency & Percentage \\
\hline $15-24$ & 1 & 1.19 \\
$25-34$ & 3 & 3.57 \\
$35-44$ & 20 & 23.8 \\
$45-54$ & 37 & 44 \\
$55-64$ & 17 & 20.2 \\
$>65$ & 6 & 7.14 \\
\hline
\end{tabular}

Table I shows the age distribution of study population.Mean age of our study group was 49.6 \pm 10.1 (28-85). Distribution of subjects on the basis of age group showed 57 (67.8\%) fell between 3554 years. Only $6(7.14 \%)$ had age more than 65 and $1(1.19 \%)$ below 35 years.

Table-II

Occupational distribution of study population $(N=85)$

\begin{tabular}{lcc}
\hline Occupation & Frequency & Percent \\
\hline Housewife & 58 & 69 \\
Manual worker /Trader & 5 & 5.9 \\
Teacher & 6 & 7.14 \\
Writer /clerk & 4 & 4.76 \\
Driver & 2 & 2.38 \\
Student & 1 & 1.19 \\
Nurse & 7 & 8.33 \\
\hline
\end{tabular}

Of the total 85 subjects 58 (69\%) were housewife. Teacher and nurse constituted $6(7.14 \%)$ and 7 $(8.33 \%)$ respectively of the subjects of the total . Other patients were white-collar workers having clerical work, computer job, trader and driver. Table II depicts the occupational distribution of study population.

Table-III

Clinical grading of CTS severity of study population $(N=85)$

\begin{tabular}{llcl}
\hline $\begin{array}{l}\text { Clinical grading } \\
\text { of CTS severity }\end{array}$ & $\begin{array}{l}\text { Clinical history } \\
\text { and objective } \\
\text { findings }\end{array}$ & Frequency & Percent \\
\hline Mild & Paresthesia & 46 & 54.56 \\
Moderate & $\begin{array}{l}\text { Sensory loss } \\
\text { Severe }\end{array}$ & 14 & 16.66 \\
& $\begin{array}{l}\text { Disruption of sleep } \\
\text { due to pain }\end{array}$ & 20 & 28.58 \\
& $\begin{array}{l}\text { Thenar muscles } \\
\text { atrophy and/or } \\
\text { weakness }\end{array}$ & 4 & \\
& & & \\
\hline
\end{tabular}

Table III shows the clinical grading based on associated clinical history and objective finding as per Mackinnson's classification.burden of CTS symptoms of study population. $54.56 \%$ patients were categorized as mild CTS with Paresthesia and $16.66 \%$ had moderate CTS with sensory loss 
in median nerve distribution clinically. $28.58 \%$ patients experienced symptoms of severe CTS with frequent disruption of sleep due to pain and Thenar muscles atrophy and/or weakness in $4 \%$ cases.

The diagnosis of CTS was confirmed and study subjects were graded according to the severity on the basis of The Canterbury NCS electrophysologic grading scale. Grading on the basis of The Canterbury NCS electrophysologic grading scale was shown in Table IV. Eighty-two (96.5\%) out of 85 had moderate to severe grade of CTS. But grade 3,4 and 5 constituted the major bulk of it 80 $(94.14 \%)$. Of the $3(3.6 \%)$ male 1 (one) each had grade 3, 4 and 5 CTS according to the Canterbury NCS electrophysologic grading scale.

\section{Table-IV}

Distribution of different grades of severity of CTS on the basis of The Canterbury NCS electrophysologic grading scale in study population $(N=85)$

\begin{tabular}{lcc}
\hline Grade & Number & Percent \\
\hline Grade 0 (normal) & 0 & 0 \\
Grade 1 (very mild) & 0 & 0 \\
Grade 2 (Mild ) & 3 & 3.5 \\
Grade 3 (Moderate) & 26 & 30.5 \\
Grade 4 (Severe) & 25 & 29.4 \\
Grade 5 (Very severe) & 29 & 34.1 \\
Grade 6 (Extremely severe) & 2 & 2.39 \\
\hline
\end{tabular}

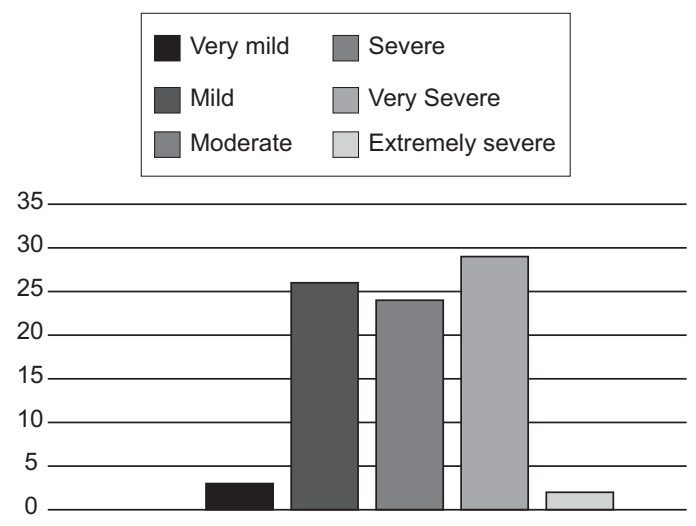

Fig.-1: Distribution of different grades of severity of carpal tunnel syndrome (CTS) in 100 hands

Figure 1 shows percentages and number of hands placed in different grades of severity according to electro diagnostic criteria. Grade V (Very severe)
CTS was the most frequent one $(34.1 \%)$ followed by Grade 3 (moderate) in $30.5 \%$ of hands.
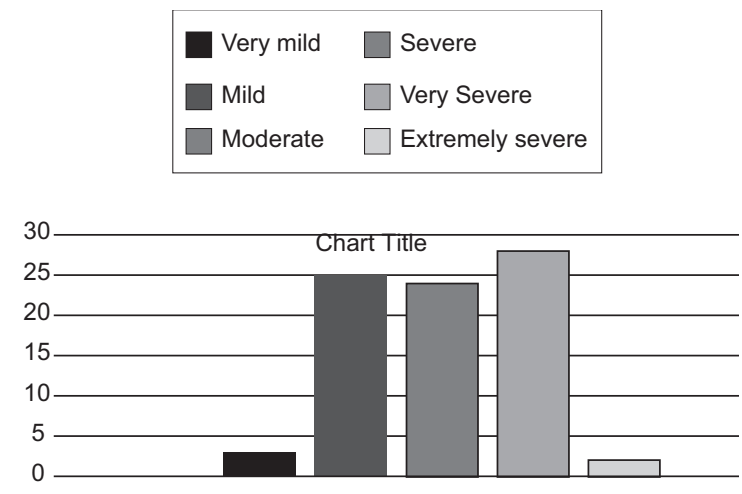

Fig.-2: Frequency of different grades of severity of CTS in female patients

Figure2 shows the frequency of different grades of CTS among female population of our study group.

Involvement of hands with CTS of the study subjects was shown in Table 5 . In our study population 22 (26.19\%) had bilateral disease and rest $(73.81 \%)$ had unilateral disease. Right hand (dominant) was more frequently involved $(n=68)$ compared to left $(n=13)$ with ratio of $5: 1$ Twenty two cases showed involvement of both hands. Right and left hand was involved in 65 and 13 cases respectively. Grade 3 damage according to the Canterbury NCS electrophysiological grading scale was present in 19, 4 and 7 in right, left and both hands respectively. Grade 4 damage was present in 18, 2 and 5 hand respectively. Grade 5 damage was present in 24,7 and 8 hand respectively.

Table-V

Distribution of unilateral (right /left) or bilateral disease in 100 hands

\begin{tabular}{lcccc}
\hline Grade & $\begin{array}{c}\text { Number of } \\
\text { hands }\end{array}$ & $\begin{array}{c}\text { Right } \\
\text { hand }\end{array}$ & $\begin{array}{c}\text { Left } \\
\text { hand }\end{array}$ & $\begin{array}{c}\text { Both } \\
\text { hands }\end{array}$ \\
\hline Grade 0 (normal) & 0 & 0 & & \\
Grade 1 (very mild) & 0 & 0 & 0 & 0 \\
Grade 2 (Mild ) & 3 & 3 & 0 & 0 \\
Grade 3 (Moderate) & 30 & 19 & 4 & 7 \\
Grade 4 (Severe) & 25 & 18 & 2 & 5 \\
Grade 5 (Very severe) & 38 & 24 & 7 & 8 \\
Grade 6 (Extremely severe) & 2 & 2 & 0 & 0 \\
& 100 & 65 & 13 & 22 \\
\hline
\end{tabular}




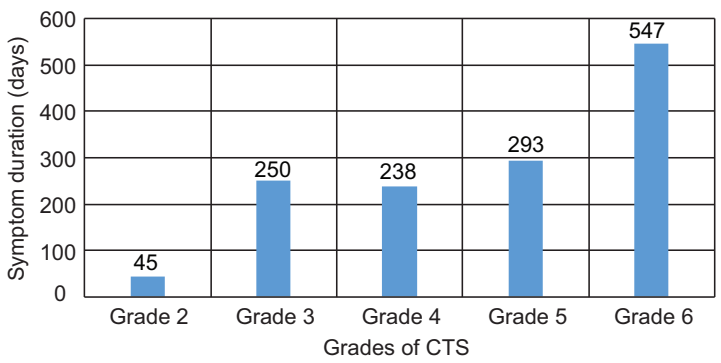

Fig.-3: Distribution of different grades of CTS in study population $(\mathrm{N}=84)$ according to duration of symptoms

Correlation between duration symptoms of CTS and different grades of CTS according to electrodiagnostic criteria was sought and in this study and is shown in Figure 3 . The mean duration of symptoms was 45 days in case of mild disease, 250 days in case of moderate disease, 238 days in case of severe disease, 293 days in case of very severe and 547 days in case of extremely severe disease.

\section{Discussion:}

Carpal tunnel syndrome is a common disorder among adults. Depending on its definition, the estimated prevalence of CTS in the general population is 1 to 5 percent ${ }^{9}$. CTS is more frequent in women ( 0.7 to 9.2 percent) than in men ( 0.4 to 2.1 percent) ${ }^{10}$. In a larger study, the female to male ratio for CTS prevalence was approximately 3 to $1^{11}$. A marked female predominance was observed in this current study group of diabetic patients with CTS. But again these results are based on single centre study, further multi-centered studies in this could suggestthe actual prevalence and female to male ratio.

Mean age of this study group was $49.6 \pm$ 10.1 years. The mean age at diagnosis was 46 (range 16-96) years in large case-control study using the UK General Practice Research Database $^{12}$. In the current study it was observed that incidence of carpal tunnel syndrome in diabetic population showed a positive correlation with increasing age of the patient,as $71.34 \%$ of our patients were above the age of 45.Similar results were observed in other studies ${ }^{13}$.
There is increasing evidence suggesting that several occupational and biomechanical factors are associated with CTS. Occupational factors that have been proposed to cause or aggravate CTS include repetitive hand and wrist use, forceful hand and wrist use, work with vibrating tools, sustained wrist or palm pressure, prolonged wrist extension and flexion and use of hands in cold temperatures. In our study that majority of sufferers were housewives. This result is in favour of some other study results ${ }^{14}$. whereas some studies showed Carpal tunnel syndrome may be present in up to $42 \%$ in workers in certain occupations (e.g., poultry processing) and has annual incidence of 193 per 100,000 in all women ${ }^{15}$. Other high -risk occupations are computer professionals ${ }^{16}$, assembly line workers, concert pianists and construction workers with vibrating power tools ${ }^{17}$.

CTS is a clinical diagnosis that is suspected when the characteristic symptoms of tingling or numbness affects the first 3 fingers and the radial aspect of the 4 th finger and specific provocative tests of the hand such as Phalen's sign and, Tinel's signs are found. Weakened grip and difficulty performing fine motor tasks may occur in more advanced carpal tunnel syndrome. On physical examination, careful observation may reveal sensory loss of median nerve innervated area , mild flattening of the thenar eminence or frank atrophy ${ }^{18}$. The current study population was clinically diagnosed and categorized into 3 grades of severity. Other studies observed correlation of clinical severity with functional disability by The Quick Disabilities of Arm, Shoulder and Hand (QDASH) scale and found a statistically significant positive relationship between QDASH results and clinical severity $(r=0.43 ; p=0.0001)^{19}$.

NCS is one of the most sensitive and specific tools for diagnosing CTS. Optional testing includes needle EMG of C5 toT1 muscles (to exclude cervical radiculopathyas a contributing factor) ${ }^{20}$.

Though there are different ways of expressing the severity of carpal tunnel syndrome (CTS) we used the Canterbury NCS Severity Scale for CTS; which are largely independent of the exact normal values used in any given laboratory and demonstrate a highly significant linear relationship between the 
neurophysiological grading and a numerical score derived from the clinical history ${ }^{21}$. The comparison with symptom score reveals a strong linear relationship and shows that the neurophysiological ranking does correspond to a clinical variable, in agreement with other studies ${ }^{22}$.

In the current study dominant hand was frequently involved however frequency of bilateral lesion in electrophysiological studies was $26.19 \%$. In other studies bilateral manifestation is more common than unilateral $(60 \%)$, but significantly more often begins or is more strongly expressed in the dominant hand ${ }^{23}$. Our results were influenced by impression of the referring physician and not entirely by patients complaints.

The current study showed a trend towards more severe electrophysiologic CTS than in those reported in the literature ${ }^{24,25}$ Yazdanpanah $\mathrm{P}$ et al ${ }^{26}$ in their study conducted on both pregnant and nonpregnant females found that out of sixty-one nonpregnant women who had CTS, $73.6 \%$ had mild, $20.8 \%$ had moderate and $5.6 \%$ had severe CTS. In our observation of 100 hands , $3 \%$ had mild ,30\% moderate , $25 \%$ severe , $38 \%$ very severe and $2 \%$ had extremely severe CTS as classified electro physiologically. The high frequency of moderate to very severe grade CTS might be due to the fact that we recruited our patients who were referred for electro diagnostic studies for their symptoms, while in Iranian study the study sample was from general population.

A positive correlation between age and severity of nerve conduction abnormality, was noted by many authors ${ }^{27}$, and might be contributory factor in our study too. Several mechanisms including mechanical compression and microvascular insufficiency may be suggested to cause severer CTS in diabetic patients ${ }^{28}$.

This observational study showed duration of disease influences electrophysiological severity of CTS. A study to find relation of symptom severity and functional status of CTS patients with electrophysiological findings using The Quick Disabilities of the Arm, Shoulder, and Hand (QuickDASH) questionnaire concluded that QDASH results were high in patients whose duration of the disease was $>6$ years and in patients with severe clinic symptoms. Also, in a correlation analysis, a positive correlation between disease duration and clinical severity with QDASH results. However, no relationship between the electrophysiological level and QDASH results was found ${ }^{20}$.

In this current observation nerve conduction studies have been used as a quantitative measure of severity of CTS and can be considerable assistance to anyone attempting to compare different studies on CTS. Our study also has some limitations. The natural history of CTS in Diabetic population is not well defined. Study including more patients with CTS, with baseline and follow-up data on symptoms and neurophysiologic parameters are needed to predict prognostic factors. Multicentre based studies in general population are required to know about the prevalence of disease and health cost burden in Bangladesh.

\section{Conclusion:}

Data demonstrated female preponderance of the diabetic CTS cases of middle aged housewives. Proportional graded deterioration of electrophysiological parameters along with the clinical severity grades highlights the fact that NCS provide additional and independent objective evidence in the diagnosis and severity assessment of CTS and plausibly has important role in prioritizing treatment plan. Study also shows that diabetic patients with CTS become symptomatic within short duration of disease ;so early recognition of symptoms and prompt referral for electrophysiological testing is recommended.

\section{References:}

1. Alfonso C, Jann S, Massa R, Torreggiani A. Diagnosis treatment andfollow-up of the carpal tunnel syndrome: a review. Neurolog Sci. 2010;31(3): 243-52.

2. Becker J, Nora DB, Gomes I, Stringari FF, Seitensus R, Panosso JS, et al.An evaluation of gender, obesity, age and diabetes mellitus as riskfactors for carpal tunnel syndrome. Clin Neurophysiol 2002; 113 :1429-34. 
3. Palmer K. Carpal tunnel syndrome: The role of occupational factors.BestPract Res Clin Rheumatol. 2011; 25(1): 15-29.

4. Srikanteswara PK, Cheluvaiah JD, Agadi JB, Nagaraj K. The Relationship between Nerve Conduction Study and Clinical Grading of Carpal Tunnel Syndrome.J Clin Diagn Res. 2016 Jul;10(7):OC13-8.

5. Jarvik JG, Comstock BA, Kliot M, Turner JA, Chan L, Heagerty PJetal. Surgery versus nonsurgical therapy for carpal tunnel syndrome: a randomised parallel-group trial. Lancet 2009;374(9695):1074

6. Huisstede BM, Fride'n J, Coert JH, et al. Carpaltunnel syndrome: hand surgeons, hand therapists, and physical medicine andrehabilitation physicians agree on amultidisciplinary treatment guideline Vresultsfrom the European HANDGUIDE Study. ArchPhys Med Rehab 2014;95(12): $2253 Y 2263$.

7. Chowdhury RN, Hussain ME, Islam MN, Hosen M, Khan AFMAM, MdFerdousMian.An audit of patients referred for nerve conduction study in a tertiary care hospital in Bangladesh.J Dhaka Med Coll. 2014; 23(1): 102-108.

8. Islam MN, Chowdhury MSJH,Chowdhury MTI,Chowdhury AH, Islam M, Hossain E etal .Identification of common risk factors associated with Carpal tunnel Syndrome. Bangladesh J Neuroscience 2013; Vol 29(2):70-78

9. AhmedA, IslamM R, RahmanHZ, BhuiyanMM. Efficacy of Local Corticosteroid in Idiopathic Carpal Tunnel Syndrome: A Randomized Controlled Trial. Bangladesh J Neuroscience 2012; Vol. 28 (1): 10-15

10. Atorshi I, Gummenson C, Johnsson R, Ornstein E, Ranstam J, Rosen I etal. Prevalence of carpal tunnel syndrome in a general population.JAMA 1999; 282(2): 153-8.

11. Stevens JC, Witt JC, Smith BE, Weaver AL. The frequency of carpal tunnel syndrome in computer users at a medical facility. Neurology 2001; 56:1568.

12. Latinovic R, Gulliford MC, Hughes RA. Incidence of common compressive neuropathies in primary care. $J$ $\mathrm{N}$ e u $\mathrm{r}$ o I $\mathrm{N}$ e u $\mathrm{r}$ o $\mathrm{s}$ u $\mathrm{r} g$ Psychiatry. 2006;77(2):263-265.

13. Claire BurtonLinda $S$ Chesterton,Graham Davenport .Diagnosing and managing carpal tunnel syndrome in primary careBr $\mathrm{J}$ Gen Pract. 2014 May; 64(622): 262-263.

14. Kouyoumdjian JA, Zanetta DM, Morita MP. Evaluation of age, body mass index, and wrist index as risk factors for carpal tunnel syndrome severity. Muscle Nerve 2002; 25:93.

15. Nehete SR, Raut BB, Hiremath AS, Thatte RM. A study the various clinical and electrophysiological parameters of severity of carpal tunnel syndrome, their correlation with post-operative recovery. Indian J Plast Surg. 2017 Sep-Dec;50(3):260-265.

16. Musolin K, Ramsey JG, Wassell JT, Hard DL. Prevalence of carpal tunnel syndrome among employees at a poultry processing plant. ApplErgon 2014; 45(6):1377Y1383.

17. Ali KM, Sathiyasekaran BWC. Computer professionals and carpal tunnel syndrome (CTS). Int J OccupSafErgon 2006; 12: 319-25.

18. Kozak A, Schedlbauer G, Wirth T, et al. Association between work-related biomechanical risk factors and the occurrence of carpal tunnel syndrome: an overview of systematic reviews and a meta-analysis of current research. BMC Musculoskelet Disord 2015; 16:231.

19. Kaplan SJ, Glickel SZ, Eaton RG .Predictive factors in the non-surgical treatment of carpal tunnel syndrome.J Hand Surg $\mathrm{Br}$. 1990;15(1):106

20. Yasemin E, Ne_e G Y, , Selim S C. The relationship between QDASH scale and 
clinical, electrophysiological findings in carpal tunnel syndrome. Advances in Clinical and Experimental Medicine 2018;27(1):71-75

21. American Association of Electro diagnostic Medicine, American Academy of Neurolog and American Academy of Physical Medicineand Rehabilitation. Practice parameter Forelectrodiagnostic studies in carpal tunnelsyndrome: summary statement. MuscleNerve 2002;25(6):918Y922.

22. You H, Simmons Z, Freivalds A, Kothari MJ, Naidu SH. Relationshipsbetween clinical symptom severity scales and nerve conduction measures in carpal tunnel syndrome. Muscle Nerve 1999;22:497-501.

23. Dec P, Zyluk A .Bilateral carpal tunnel syndrome - A review. NeurolNeurochir Pol. 2018 Jan - Feb;52(1):79-83.

24. Ali Z, Khan A, Shah SM, Zafar A. Clinical and electro-diagnostic quantification of the severity of carpal tunnel syndrome. Ann Pak Inst Med2012Sci 8: 207-212.

25. Padua L, LoMonaco M, Gregori B, Valente EM, Padua R, et al). Neurophysiological classification and sensitivity in 500 carpal tunnel syndrome hands. Acta Neurol Scand 1997; 96: 211-217.

26. Yazdanpanah P, Aramesh S, Mousavizadeh A, Ghaffari P, KhosraviZ, KhademiA et al. Prevalence and severity of carpal tunnel syndromein women. Iran J Public Health. 2012; 41: 105-110.

27. Kouyoumdjian JA. Carpal tunnel syndrome. Age, nerve conductionseverity and duration of symptomatology. ArqNeuropsiquiatr 1999;57:382-386.

28. Yoo Hwan Kim,Kyung-Sook Yang, Hanjun Kim, Hung YoulSeok, Jung Hun Lee, Myeong Hun Son et al. Does Diabetes Mellitus Influence Carpal Tunnel Syndrome?J Clin Neurol. 2017 Jul; 13(3): 243-249. 